\title{
A New Approach to Fuzzy Metric Spaces and Their Similarity-Based Construction
}

\author{
Gültekin Soylu and Mutlu Güloğlu \\ Department of Mathematics, Akdeniz University, 07058 Antalya, Turkey \\ Correspondence should be addressed to Gültekin Soylu; gsoylu@akdeniz.edu.tr \\ Received 13 January 2016; Accepted 23 March 2016 \\ Academic Editor: Hugo Leiva
}

Copyright ( 2016 G. Soylu and M. Güloğlu. This is an open access article distributed under the Creative Commons Attribution License, which permits unrestricted use, distribution, and reproduction in any medium, provided the original work is properly cited.

We introduce a space of functions which can be interpreted as a similarity-based approach to fuzzy metric spaces. The triangle inequality we propose is defined by means of a fuzzy ordering. We compare the introduced space with fuzzy metric spaces in the sense of Seikkala and Kaleva. Finally we complete the work discovering the corresponding classical as well as fuzzy topologies.

\section{Introduction}

The theory of metric spaces, first introduced by Fréchet in 1906 [1], stems from the idealization of the notion of distance. In a metric space the distance between two points of a set is represented by a single nonnegative real number. However, in many cases of physical or other scientific problems of interest, it seems to be too ideal to associate the distance between two points of a set with a single nonnegative real number. Most probably this fact inspired Menger in 1942 [2] to generalize the concept of a metric space in a statistical manner. He was modeling the distance by a probability of being less than a value. Wald [3] and later Schweizer and Sklar [4] made worthful contributions to the theory of statistical metric spaces. Motivated by these works in 1975 Kramosil and Michalek [5] introduced an equivalent notion, namely, the fuzzy metric space. They were modeling the case where the inexactness of the distance is due to incomplete information rather than randomness. A lot of work is done on this subject with small modifications especially by George and Veeramani [6] and by Gregori and Romaguera [7]. However, Kaleva and Seikkala [8] felt that (and the authors of this paper share this feeling) it is a more natural way to define the fuzzy metric space by setting the distance between two points to be a nonnegative fuzzy number. Following this latter approach, in this work we introduce the similarity-based fuzzy metric spaces. We try to model the case where the inexactness of the distance between two points is due to the indistinguishability of the measured values. Kaleva and Seikkala propose a triangular inequality on the fuzzy numbers which employs both the addition of fuzzy numbers and a crisp partial ordering between them. The fuzzy triangular inequality we propose employs the notion of fuzzy orderings rather than crisp orderings. In fact our definition of fuzzy triangular inequality is just a rendering of the crisp one to the semantics of $t$-norm fuzzy logic. This seems to be advantageous in the theoretic point of view because this new concept is in harmony with formerly defined similarity-based fuzzy concepts such as fuzzy orderings and fuzzy limits. The smooth connection between those concepts is a result of the underlying $t$-norm logic in common and is therefore promising for possible further works related to the introduced fuzzy metric. On the other hand from the practical point of view a fuzzy metric in our setting is easy to understand and very easy to construct: all you need to start with is a fuzzy set. Nevertheless the proposed approach is not completely different from its predecessors; the close relation between our proposal and the one of Kaleva and Seikkala is also presented in the work. At the end of the paper, for the sake of completeness of the work, we establish the corresponding classical and fuzzy topologies to a given similarity-based fuzzy metric.

\section{Preliminaries}

For simplicity in this paper the set of truth degrees is considered as the unit interval $[0,1]$ and is denoted by $I$. The 
set of all real numbers is shown by $\mathbb{R}$ and the symbol * will denote a left-continuous $t$-norm [9].

The support of a fuzzy subset $\mu$ of a set $X$ is defined as the crisp set, $\{x \in X: \mu(x)>0\}$, and is denoted by $\operatorname{Supp}(\mu)$.

The core of a fuzzy subset $\mu$ of a set $X$ is defined as the crisp set, $\{x \in X: \mu(x)=1\}$, and is denoted by $\operatorname{Core}(\mu)$.

A fuzzy subset $\mu$ of a set $X$ is called to be normal if there exists $x \in X$ with $\mu(x)=1$ (i.e., $\operatorname{Core}(\mu)$ is nonempty).

Let $\mu$ be a fuzzy subset of the space $\mathbb{R}^{n}$; then we say that $\mu$ is fuzzy convex [10] if $\mu\left(\lambda x_{1}+(1-\lambda) x_{2}\right) \geq \min \left(\mu\left(x_{1}\right), \mu\left(x_{2}\right)\right)$, for all $x_{1}, x_{2} \in \mathbb{R}^{n}$, and all $\lambda \in[0,1]$.

Suppose that $X$ is a topological space, $x_{0}$ is a point in $X$, and $f: X \rightarrow \mathbb{R}$ is a function. We say that $f$ is upper semicontinuous at $x_{0}$ if for every $\varepsilon>0$ there exists a neighborhood $U$ of $x_{0}$ such that $f(x) \leq f\left(x_{0}\right)+\varepsilon$ for all $x \in U$.

A fuzzy number [11] $\mu$ is a fuzzy subset of the real line $\mathbb{R}$, that is, a mapping $\mu: R \rightarrow I$ which is normal, fuzzy convex, and upper semicontinuous, and $\operatorname{Supp}(\mu)$ is bounded. The set of all fuzzy numbers will be denoted by $\mathscr{F}$.

The $\alpha$-level sets of a fuzzy number $\mu$ are denoted by $[\mu]_{\alpha}$ and are defined by $[\mu]_{\alpha}=\{r \in \mathbb{R}: \mu(r) \geq \alpha\}, 0<\alpha \leq 1$.

A fuzzy subset of $\mathbb{R}$ is fuzzy convex if and only if each of its $\alpha$-level sets is a convex set in $\mathbb{R}$. The upper semicontinuity condition guarantees that the $\alpha$-level set of a fuzzy number is a closed interval $\left[a^{\alpha}, b^{\alpha}\right]$. One should note that the intervals and real numbers can be embedded in $\mathscr{F}$ since every interval $[a, b]$ can be represented by a fuzzy number $\overline{[a, b]}$ defined by

$$
\overline{[a, b]}(t)= \begin{cases}1, & a \leq t \leq b, \\ 0, & \text { otherwise }\end{cases}
$$

and, similarly, every real number $r$ can be represented by a fuzzy number $\bar{r}$ defined by

$$
\bar{r}(t)= \begin{cases}1, & t=r, \\ 0, & t \neq r .\end{cases}
$$

If $\mu(r)=0$ for all $r<0$ then $\mu$ is called a nonnegative fuzzy number and the set of all nonnegative fuzzy numbers will be denoted by $\mathscr{G}$.

For a choice of three real numbers $a_{1}, a_{2}$, and $a_{3}$ with $a_{1}<a_{2}<a_{3}$ a triangular fuzzy number $\mu$ is represented by $\left(a_{1}, a_{2}, a_{3}\right)$ and is defined by

$$
\mu(x)=\left\{\begin{array}{ll}
0 & x<a_{1}, \\
\frac{x-a_{1}}{a_{2}-a_{1}} & a_{1} \leq x \leq a_{2}, \\
\frac{a_{3}-x}{a_{3}-a_{2}} & a_{2} \leq x \leq a_{3}, \\
0 & a_{3}<x,
\end{array} \quad \forall x \in \mathbb{R} .\right.
$$

A map $E: X \times X \rightarrow I$ is called a $*$-similarity relation on $X$ iff the following three axioms are satisfied:

(E.1) $E(x, x)=1, \forall x \in X$,

(E.2) $E(x, y)=E(y, x), \forall x, y \in X$,

(E.3) $(x, y) * E(y, z) \leq E(x, z), \forall x, y, z \in X$.
A *-similarity relation $E$ on $X$ is said to be separated (i.e., a *-equality relation) iff the implication $E(x, y)=1 \Rightarrow x=y$ is satisfied for all $x, y \in X$.

Although fuzzy orderings related to an underlying *similarity relation were first defined by Höhle and Blanchard [12] and developed by researchers like Bělohlávek [13], we will use throughout this paper Bodenhofer's contribution [14, 15] to this subject.

Definition 1. Let $E$ be a $*$-similarity on a set $X$; a fuzzy relation $\preccurlyeq \in I^{X \times X}$ is called an $E$-partial ordering on $X$ if it satisfies the following properties:

$$
\begin{aligned}
& (\text { EPO.1) } E(x, y) \leq \preccurlyeq(x, y), \forall x, y \in X . \\
& (\text { EPO.2) } \preccurlyeq(x, y) * \preccurlyeq(y, x) \leq E(x, y), \forall x, y \in X . \\
& (\text { EPO.3) } \preccurlyeq(x, y) * \preccurlyeq(y, z) \leq \preccurlyeq(x, z), \forall x, y, z \in X .
\end{aligned}
$$

An $E$-partial ordering on $X$ is called a strongly linear $E$-ordering if it satisfies.

$$
(\text { EPO.4) } \preccurlyeq(x, y) \vee \preccurlyeq(y, x)=1, \forall x, y \in X \text {. }
$$

Definition 2 . Let $\preceq$ be a crisp ordering on a set $X ; *$-similarity $E$ is called compatible with $\preceq$ if and only if the following implication holds for all $x, y, z \in X$ :

$$
\begin{gathered}
x \preceq y \preceq z \Longrightarrow \\
{[E(x, z) \leq E(y, z)] \wedge[E(x, z) \leq E(x, y)] .}
\end{gathered}
$$

Definition 3. Let $\circ$ be a binary operation on $X$. A *-similarity $E$ is called invariant under $\circ$ if it satisfies the following condition for all $x, y, z \in X$ :

$$
E(x, y)=E(x \circ z, y \circ z), \quad \forall x, y, z \in X .
$$

Example 4. Let $X$ be the real line and let the binary operation - be the addition. The relations $E$ defined by $E(x, y)=$ $\exp (-|x-y|)$ and $E(x, y)=\max \{0,1-|x-y|\}$ are *similarities invariant under + , where $*$ is the product $t$-norm and the Lukasiewicz $t$-norm, respectively.

In [14] also the following important theorem is proved.

Theorem 5. $\preccurlyeq \in I^{X \times X}$ is a strongly linear E-ordering if and only if there exists a linear ordering $\preceq$ on $X$ that is compatible with $E$ such that $\preccurlyeq$ can be represented as

$$
\preccurlyeq(x, y)=\left\{\begin{array}{ll}
1 & x \preceq y, \\
E(x, y) & \text { otherwise, }
\end{array} \quad \forall x, y \in X .\right.
$$

Throughout the text, whenever a $*$-similarity $E$ on $X$ compatible with a linear ordering $\preceq$ on $X$ is given the corresponding $E$-ordering will be assumed to be the one defined in (6).

\section{Similarity-Based Fuzzy Metric}

The concept of probabilistic metric spaces [4] was introduced to mathematics as a tool which deals with situations where 
the distance between two points is uncertain. The uncertainty was assumed to be caused by randomness. But later trying to deal with uncertainty caused by incomplete information in [8] the authors have introduced the following definition for a fuzzy metric space.

Let $X$ be a nonempty set and $d$ a mapping from $X \times X$ into $\mathscr{G}$ and let the mappings $L, R: I \times I \rightarrow I$ be symmetric, nondecreasing in both arguments and satisfy $L(0,0)=0$ and $R(1,1)=1$. Denote

$$
\begin{aligned}
& {[d(x, y)]_{\alpha}=\left[\lambda_{\alpha}(x, y), \rho_{\alpha}(x, y)\right]} \\
& \qquad \text { for } x, y \in X, 0<\alpha \leq 1 .
\end{aligned}
$$

The quadruple $(X, d, L, R)$ is called a fuzzy metric space and $d$ a fuzzy metric, if

(F1) $d(x, y)=\overline{0} \Leftrightarrow x=y$,

(F2) $d(x, y)(r)=d(y, x)(r)$ for all $x, y \in X$, and all $r \in \mathbb{R}$,

(F3) for all $x, y, z \in X$,

(a) $d(x, y)(s+t) \geq L(d(x, z)(s), d(z, y)(t))$

whenever $s \leq \lambda_{1}(x, z), t \leq \lambda_{1}(z, y)$, and $s+t \leq$ $\lambda_{1}(x, y)$,

(b) $d(x, y)(s+t) \leq R(d(x, z)(s), d(z, y)(t))$

whenever $s \geq \lambda_{1}(x, z), t \geq \lambda_{1}(z, y)$, and $s+t \geq$ $\lambda_{1}(x, y)$.

In this definition axiom (F3) is a generalization of the triangular inequality and typical examples for the mappings $L$ and $R$ are $t$-norms and $t$-conorms, respectively. For the particular choices $L=\operatorname{Min}$ and $R=\operatorname{Max}(\mathrm{F} 3)$ is equivalent to

$$
d(x, y) \leqslant d(x, z) \oplus d(z, y),
$$

where $\oplus$ is Zadeh's Extension [16] of addition to fuzzy numbers and $\leqslant$ is a crisp partial ordering on fuzzy numbers [17]. An example for fuzzy metric spaces will be given in the sequel right after Theorem 10 . We propose the following definition.

Definition 6 . Let $E$ be a $*$-equality on $\mathbb{R}$ and $\preccurlyeq$ an $E$-ordering on $\mathbb{R}$. A fuzzy set $\delta$ in $X \times X \times \mathbb{R}$ is called a similarity-based fuzzy metric if it satisfies the following properties:

(FM1) $r<0 \Rightarrow \delta(x, y)(r)<1, \forall x, y \in X$,

(FM2) $\delta(x, y)(r)=\delta(y, x)(r), \forall x, y \in X, \forall r \in \mathbb{R}$,

(FM3) $\delta(x, y)(0)=1 \Leftrightarrow x=y, \forall x, y \in X$,

(FM4) $\delta(x, y)(a) * \delta(y, z)(b) * \delta(x, z)(c) \leq \preccurlyeq(c, a+b), \quad \forall x, y$, $z \in X, \forall a, b, c \in \mathbb{R}$.

The quadruple $(X, E, \preccurlyeq, \delta)$ will be called a similarity-based fuzzy metric space.

This definition is a slight modification of the one in [18]. The axioms given here are the many valued counterparts of the well-known metric axioms. Among these axioms (FM1), (FM2), and (FM3) are direct transformations whereas (FM4) is the $t$-norm logic counterpart of the following reformulation of the triangular inequality.

If the distances between $x, y$ and $y, z$ and $x, z$ are $a, b$, and $c$, respectively, then $c$ should be less than or equal to $a+b$. Analogously (FM4) can be interpreted as follows: if it is true to a certain degree that the distances between $x, y$ and $y, z$ and $x, z$ are $a, b$, and $c$, respectively, then it should be true at least to the same degree that $c$ is less than or equal to $a+$ $b$. One should note that the $E$-ordering plays an important role in this last axiom: the fact that in the two-valued case (FM4) will be equivalent to the classic triangular inequality is a consequence of the fact that in this case the E-ordering becomes a crisp ordering.

In the sequel we will denote $[\delta(x, y)]_{\alpha}=\left[\lambda_{\alpha}(\delta(x, y))\right.$, $\left.\rho_{\alpha}(\delta(x, y))\right]$ for $x, y \in X, 0<\alpha \leq 1$.

In the following theorem we establish a connection between fuzzy metric spaces and metric spaces. The proof will be in a similar fashion to the proof of Theorem 3.2 in [8] in order to show the close relation between the two approaches.

Definition 7. A uniformity for a set $X$ is a nonempty family $\mathcal{U}$ of subsets of $X \times X$ which satisfies the following conditions:

(i) Each member of $\mathcal{U}$ contains the diagonal $\Delta=\{(x, x)$ : $x \in X\}$.

(ii) If $U \in \mathcal{U}$, then $U^{-1} \in \mathcal{U}$.

(iii) If $U \in \mathcal{U}$, then $V \circ V \subset U$ for some $V$ in $\mathcal{U}$, where $V \circ V$ denotes the composite of $V$ with itself.

(iv) If $U, V \in \mathcal{U}$, then $U \cap V \in \mathcal{U}$.

(v) If $U \in \mathcal{U}$ and $U \subset V \subset X \times X$, then $V \in \mathcal{U}$.

If $\mathcal{U}$ is a uniformity, then the pair $(X, \mathcal{U})$ is called uniform space [19].

Theorem 8. A nonempty family $\mathscr{B}$ of subsets of $X \times X$ is a base for some uniformity for $X$ if and only if one has the following:

(i) Each member of $\mathscr{B}$ contains the diagonal.

(ii) If $U \in \mathscr{B}$, then $U^{-1}$ contains a member of $\mathscr{B}$.

(iii) If $U \in \mathscr{B}$, then $V \circ V \subset U$ for some $V$ in $\mathscr{B}$.

(iv) Intersection of two members of $\mathscr{B}$ contains a member [19].

A space with a uniform topology is a Hausdorff space iff $\bigcap\{U: U \in \mathcal{U}\}$ is the diagonal $\Delta$. In this case $(X, \mathcal{U})$ is said to be Hausdorff [19].

Theorem 9. Let $(X, E, \widetilde{\preccurlyeq}, \delta)$ be a similarity-based fuzzy metric space, where $E$ is a *-equality on $\mathbb{R}$ compatible with $\leq$, $\preccurlyeq$ is the corresponding E-ordering, and Core $\delta(x, y)$ is a nonempty set for any choice of $x, y$ in $X$. Then the family $\mathcal{U}=\{U(\varepsilon): \varepsilon>0\}$ of sets

$$
U(\varepsilon)=\left\{(x, y) \in X \times X: \rho_{1}(\delta(x, y))<\varepsilon\right\}
$$

forms a basis for a Hausdorff uniformity on $X \times X$ and the corresponding uniform topology is metrizable. 
Proof. To see that the family of the sets $U(\varepsilon)$ forms a basis for a Hausdorff uniformity on $X \times X$, we will show that the sets $U(\varepsilon)$ satisfy the axioms for a basis for a Hausdorff uniformity $[19$, pages 174-180] as follows.

We keep in mind that Core $\delta(x, y)$ is nonempty and therefore $\rho_{1}(\delta(x, y))$ always exists.

(i) Let $U(\varepsilon) \in \mathscr{U}$. By (FM3) we obtain that $\rho_{1}(\delta(x, x))=$ $0<\varepsilon$ which implies $(x, x) \in U(\varepsilon)$ and thereby

$$
\{(x, x): x \in X\} \subset U(\varepsilon) .
$$

(ii) Let $(x, y) \in U(\varepsilon) \in \mathcal{U}$. Since this means that $\rho_{1}(\delta(x$, $y))<\varepsilon$ and by (FM2) $\delta$ is symmetric we get $\rho_{1}(\delta(y, x))<\varepsilon$. We conclude that $U(\varepsilon)$ is symmetric.

(iii) Let $U(\varepsilon) \in \mathcal{U}$. On the other hand let $(x, y),(y, z) \in$ $U(\varepsilon / 2)$. So we have $\rho_{1}(\delta(x, y))<\varepsilon / 2$ and $\rho_{1}(\delta(y, z))<\varepsilon / 2$ and therefore $\rho_{1}(\delta(x, y))+\rho_{1}(\delta(y, z))<\varepsilon$.

Let $\rho_{1}(\delta(x, z))=c$; by (FM4) we obtain $c \leq \rho_{1}(\delta(x, y))+$ $\rho_{1}(\delta(y, z))<\varepsilon$ and hence $(x, z) \in U(\varepsilon)$. We conclude that $U(\varepsilon / 2) \circ U(\varepsilon / 2) \subset U(\varepsilon)$.

(iv) Let $U\left(\varepsilon_{1}\right), U\left(\varepsilon_{2}\right) \in \mathcal{U}$. Let $(x, y) \in U\left(\min \left\{\varepsilon_{1}, \varepsilon_{2}\right\}\right)$ which implies $\rho_{1}(\delta(x, y))<\min \left\{\varepsilon_{1}, \varepsilon_{2}\right\}$ and therefore $\rho_{1}(\delta(x$, $y))<\varepsilon_{1}$ and $\rho_{1}(\delta(x, y))<\varepsilon_{2}$. Hence $(x, y) \in U\left(\varepsilon_{1}\right)$ and $(x, y)$ $\in U\left(\varepsilon_{2}\right)$. We conclude that $U\left(\min \left\{\varepsilon_{1}, \varepsilon_{2}\right\}\right) \subset U\left(\varepsilon_{1}\right) \cap U\left(\varepsilon_{2}\right)$.

Finally for being Hausdorff we observe the following.

Let $x \neq y$; by (FM1) and (FM3) we have $\rho_{1}(\delta(x, y))>0$. Setting $\varepsilon=\rho_{1}(\delta(x, y))$, we obtain $(x, y) \notin U(\varepsilon)$. We deduce that the intersection of the members of the family $\mathcal{U}$ is the diagonal of $X \times X$.

Now since for a nonnegative sequence $\left\{\varepsilon_{n}\right\}$ converging to 0 the family $\{U(\varepsilon)\}$ is a countable basis for the Hausdorff uniformity of discourse, the metrizability of the corresponding uniform topology follows [19, page 186].

The following theorem states the construction of a similarity-based fuzzy metric on a metric space, where $\mathbb{R}$ is equipped with a *-equality.

Theorem 10. Let $(X, d)$ be a metric space, $E$ a $*$-equality on $\mathbb{R}$ compatible with $\leq$, invariant under + , and $\preccurlyeq$ the corresponding $E$-ordering on $\mathbb{R}$. The fuzzy relation $\delta_{E}$ defined by

$$
\delta_{E}(x, y)(r)=E(d(x, y), r), \quad \forall x, y \in X, \forall r \in \mathbb{R},
$$

is a similarity-based fuzzy metric on $X$.

Proof. We will show that $\delta_{E}$ satisfies the properties (FM1)(FM4).

(FM1) Let $x, y \in X$ and $r<0$. Suppose that $\delta_{E}(x, y)(r)=1$. This would yield $E(d(x, y), r)=1$ and $d(x, y)=r<$ 0 since $E$ is a $*$-equality. So $\delta_{E}(x, y)(r)<1$.

(FM2) Let $\delta_{E}(x, y)(r)=E(d(x, y), r)=E(d(y, x), r)=$ $\delta_{E}(y, x)(r), \forall x, y \in X, \forall r \in \mathbb{R}$.

(FM3) Let $\delta_{E}(x, y)(0)=1$; then by definition $E(d(x, y), 0)=$ 1 and since $E$ is a $*$-equality we have $d(x, y)=0$ and this implies $x=y$. Conversely, let $x=y$ which implies $d(x, y)=0$ and $E(d(x, y), 0)=1$, which in return by definition yields $\delta_{E}(x, y)(0)=1$.
(FM4) In order to see that $\delta_{E}$ satisfies (FM4) it is sufficient to investigate the case where $a+b<c$ since otherwise the RHS of the inequality would be equal to 1 .

The rest of the proof consists of some subcases.

(i) Suppose that $d(x, y) \leq a, d(y, z) \leq b$, and $d(x, z) \leq c$. Keeping in mind that $\widetilde{\lessgtr}$ is the corresponding ordering to $E$ the inequality in (FM4) becomes

$$
\begin{aligned}
& E(d(x, y), a) * E(d(y, z), b) * E(d(x, z), c) \\
& \quad \leq E(c, a+b) .
\end{aligned}
$$

We exploit the triangular inequality for the metric function $d, d(x, z) \leq d(x, y)+d(y, z)$, and obtain the inequality, $d(x, z) \leq a+b<c$. Now by $\leq$-compatibility of $E$ we obtain $E(d(x, z), c) \leq E(c, a+b)$ which yields the desired inequality:

$$
\begin{aligned}
& E(d(x, y), a) * E(d(y, z), b) * E(d(x, z), c) \\
& \quad \leq E(d(x, z), c) \leq E(c, a+b) .
\end{aligned}
$$

(ii) Suppose that $c \leq d(x, z)$; in this case we have

$$
a+b<c \leq d(x, z) \leq d(x, y)+d(y, z) .
$$

Since $E$ is invariant under + and is a symmetric, transitive relation, we can write the following:

$$
\begin{aligned}
& E(d(x, y), a) * E(d(y, z), b) \\
& \quad=E(d(x, y)-a, 0) * E(0, b-d(y, z)) \\
& \quad \leq E(d(x, y)-a, b-d(y, z))
\end{aligned}
$$

(we add $a+d(y, z)$ to both arguments)

$$
=E(d(x, y)+d(y, z), a+b)
$$

(assumption and the $\leq$-compatibility of $E$ )

$\leq E(c, a+b)$.

(iii) We are left with the case where $d(x, z) \leq c, a \leq$ $d(x, y)$, and $d(y, z) \leq b$ (or $b \leq d(y, z)$ and $d(x, y) \leq a$ which is a symmetric case).

(a) We assume $a+b \leq d(x, z) \leq d(x, y)+d(y, z) \leq c$. Consider

$$
\begin{aligned}
& E(d(x, y), a) * E(d(y, z), b) \leq E(d(x, y) \\
& \quad+d(y, z), a+b) \quad(\text { which was obtained before) } \\
& \quad \leq E(d(x, z), a+b) \text { (by assumption). }
\end{aligned}
$$

Therefore we can write the following:

$$
\begin{aligned}
& E(d(x, y), a) * E(d(y, z), b) * E(d(x, z), c) \\
& \quad \leq E(d(x, z), a+b) * E(d(x, z), c) \\
& \quad \leq E(c, a+b) \text { (by transitivity of } E) .
\end{aligned}
$$


(b) We assume $a+b \leq d(x, z) \leq c \leq d(x, y)+d(y, z)$. The proof is identical to (iii)(a).

(c) We assume $d(x, z) \leq a+b \leq d(x, y)+d(y, z) \leq c$.

Here again from $\leq$-compatibility of $E$ we obtain the following inequality:

$$
\begin{gathered}
E(d(x, z), c) \leq E(d(x, y)+d(y, z), c) \\
E(d(x, y), a) * E(d(y, z), b) * E(d(x, z), c) \\
\quad \leq E(d(x, y)+d(y, z), a+b) \\
\quad * E(d(x, y)+d(y, z), c) \leq E(c, a+b) .
\end{gathered}
$$

(d) We assume $d(x, z) \leq d(x, y)+d(y, z) \leq a+b \leq c$. The proof is identical to (iii)(c).

(e) Finally we assume $d(x, z) \leq a+b \leq c \leq d(x, y)+$ $d(y, z)$.

This time we make use of $\leq$-compatibility of $E$ as follows:

$$
E(d(x, z), c) \leq E(d(x, z), a+b) .
$$

Hence,

$$
\begin{aligned}
& E(d(x, y), a) * E(d(y, z), b) * E(d(x, z), c) \\
& \leq E(d(x, y)+d(y, z), a+b) \\
& \quad * E(d(x, z), a+b) \\
& \leq E(d(x, y)+d(y, z), d(x, z))
\end{aligned}
$$

(compatibility of $E$ with $\leq$ )

$\leq E(c, a+b)$

finishes the proof.

Remark 11. It is clear that Core $\delta_{E}(x, y)$ is a nonempty set for any choice of $x, y$ in $X$ and we can conclude that $\left(X, E, \widetilde{\preccurlyeq}, \delta_{E}\right)$ is metrizable.

A result of the famous representation theorem of Valverde [20] is the following.

Let $*$ be the Lukasiewicz $t$-norm and $\left\{h_{j}\right\}_{j \in J}$ a family of fuzzy sets; then

$$
E(x, y)=\operatorname{Inf}_{j \in J}\left(1-\left|h_{j}(x)-h_{j}(y)\right|\right)
$$

is a $*$-similarity relation.

Combining this result with the preceding theorem we obtain a method for constructing a similarity-based fuzzy metric just by starting with a "sample" fuzzy set. In the following example we use a triangular fuzzy number for this purpose although it is possible to choose from several other types of fuzzy numbers.

Example 12. Let $*$ be the Lukasiewicz $t$-norm and let $\left(a_{1}, a_{2}, a_{3}\right)$ be a given triangular fuzzy number. We define the family $\left\{h_{r}\right\}_{r \in \mathbb{R}}$ to contain all horizontal shifts of the given membership function. That is,

$$
\left\{h_{r}\right\}_{r \in \mathbb{R}}=\left\{\left(a_{1}+r, a_{2}+r, a_{3}+r\right): r \in \mathbb{R}\right\} .
$$

Now we construct a $*$-similarity relation $E$ by $E(x, y)=$ $\operatorname{Inf}_{j \in \mathbb{R}}\left(1-\left|h_{j}(x)-h_{j}(y)\right|\right)$. It is easy to verify that $E(x, y)=$ $\max \{1-(|x-y| / s), 0\}$, where $s=\min \left\{a_{2}-a_{1}, a_{3}-a_{2}\right\}$. It can also be observed that $E$ is a $*$-equality compatible with $\leq$ and invariant under + so we can conclude that $\delta_{E}(x, y)(r)=$ $E(d(x, y), r), \forall x, y \in X, \forall r \in \mathbb{R}$, is a similarity-based fuzzy metric.

The intersection of fuzzy metric spaces and similaritybased fuzzy metric spaces is nonempty as it is shown in the following theorem.

Theorem 13. Let $(X, d)$ be a metric space, $E$ a $*$-equality on $\mathbb{R}$ compatible with $\leq$, invariant under + , and $\preccurlyeq$ the corresponding E-ordering on $\mathbb{R}$. The fuzzy relation $\delta$ defined by

$$
\delta(x, y)(r)= \begin{cases}0, & r<0 \\ \overline{0}, & x=y \\ E(d(x, y), r), & r \geq 0, x \neq y, \\ & \forall x, y \in X, \forall r \in \mathbb{R},\end{cases}
$$

is both a similarity-based fuzzy metric and a fuzzy metric on $X$.

Proof. We will only show that the fuzzy relation $\delta$ is a fuzzy metric.

The first two properties (F1) and (F2) are easy to observe so let us prove that $\delta$ holds the third property.

We set $L=*$ and $R=\mathrm{V}$, where $\mathrm{V}$ denotes the binary operation maximum.

(i) Let $t<0$ or $s<0$. Since one of the conditions of (F3)(b) will not hold (F3)(b) drops. Let us check (F3)(a).

The conditions $s \leq \lambda_{1}(\delta(x, z))$ and $t \leq \lambda_{1}(\delta(z, y))$ of (F3)(a) naturally hold and since either $\delta(x, z)(s)=0$ or $\delta(z, y)(t)=0$ we obtain

$$
\delta(x, z)(s) * \delta(z, y)(t) \leq \delta(x, y)(s+t) .
$$

(ii) Let $0 \leq t$ and $0 \leq s$. We will investigate 2 subcases; namely, $x=z$ (or $y=z$ ) and $x \neq z$ and $y \neq z$ :

(a) Let $x=z$ (or $y=z$ which is the symmetric case). In this case $\lambda_{1}(\delta(x, z))=0$ and for (F3)(a) the assumptions imply $s=0$. The desired inequality can easily be observed since

$$
\begin{gathered}
\delta(x, z)(s) * \delta(z, y)(t)=1 * \delta(z, y)(t) \\
=\delta(z, y)(t)=\delta(x, y)(s+t) .
\end{gathered}
$$

On the other hand for (F3)(b) let $t \geq \lambda_{1}(\delta(z, y))$ and $s+t \geq \lambda_{1}(\delta(x, y))$. If $s=0$ we have $\delta(x, z)(s)=1$ and therefore

$$
\delta(x, z)(s) \vee \delta(z, y)(t)=1 \geq \delta(x, y)(s+t) .
$$


If $s>0$ we observe that if $x=y$ we have $\delta(x, z)=\overline{0}$, $\delta(z, y)=\overline{0}$, and $\delta(x, y)=\overline{0}$, and therefore

$$
\begin{aligned}
& \delta(x, z)(s) \vee \delta(z, y)(t)=\delta(z, y)(t) \\
& \quad \geq \delta(x, y)(s+t) .
\end{aligned}
$$

Meanwhile if $x \neq y$ we have $\delta(x, y)(s+t)=$ $E(d(x, y),(s+t))$; on the other hand since $d(x, y) \leq$ $t \leq t+s$ we have $E(d(x, y), t) \geq E(d(x, y),(s+t))$. Combining these two facts we observe that

$$
\begin{aligned}
\delta(x, z)(s) \vee \delta(z, y)(t)=0 \vee E(d(x, y), t) \\
\quad=E(d(x, y), t) \geq E(d(x, y),(s+t)) \\
\quad=\delta(x, y)(s+t) .
\end{aligned}
$$

(b) Let $x \neq z$ and $y \neq z$. In this case if $x \neq y$, considering that $(s+t) \geq 0$ we have

$$
\begin{aligned}
\delta(x, z)(s) & =E(d(x, z), s), \\
\delta(z, y)(t) & =E(d(z, y), t), \\
\delta(x, y)(s+t) & =E(d(x, y),(s+t)) .
\end{aligned}
$$

Since $d(x, y) \leq d(x, z)+d(z, y) \leq s+t$ and since $E$ is compatible with $\leq$ and invariant under + we have

$$
\begin{aligned}
E & (d(x, y),(s+t)) \leq E(d(x, z)+d(z, y),(s+t)) \\
& \leq E(d(x, z)+d(z, y), s+d(z, y)) \\
& =E(d(x, z), s)
\end{aligned}
$$

and similar to this

$$
\begin{aligned}
E & (d(x, y),(s+t)) \leq E(d(x, z)+d(z, y),(s+t)) \\
& \leq E(d(x, z)+d(z, y), t+d(x, z)) \\
& =E(d(z, y), t) .
\end{aligned}
$$

So we obtain the required inequality by the following:

$$
\begin{aligned}
\delta(x, z)(s) \vee \delta(z, y)(t) \\
\quad=E(d(x, z), s) \vee E(d(z, y), t) \\
\quad \geq E(d(x, y),(s+t))=\delta(x, y)(s+t) .
\end{aligned}
$$

If $x=y$ the fact that $\delta(x, y)=\overline{0}$ and $s+t \neq 0(t \geq$ $\lambda_{1}(\delta(z, y))>0$ and $\left.s \geq \lambda_{1}(\delta(x, z))>0\right)$ implies $\delta(x, y)(s+t)=0$ so the desired inequality holds and the proof is completed.

Example 14. Consider the metric space $\mathbb{R}$ with its standard metric. Let $*$ be the product $t$-norm. It is well-known that $E(x, y)=\exp (-|x-y|)$ is a $*$-equality on $\mathbb{R}$; moreover, it is easy to check that $E$ is invariant under + and compatible with the ordering on $\mathbb{R}$. Finally let $\preccurlyeq$ be the corresponding $E$-ordering. The fuzzy relation $\delta$ defined by

$$
\delta(x, y)(r)= \begin{cases}0, & r<0 \\ \overline{0}, & x=y \\ \exp (-|| x-y|-r|), & r \geq 0, x \neq y, \\ \forall x, y & \in X, \quad \forall r \in \mathbb{R},\end{cases}
$$

is an example for both a similarity-based fuzzy metric and a fuzzy metric on $\mathbb{R}$, where the functions $L$ and $R$ are chosen to be product and maximum, respectively.

Now we will try to show a connection between fuzzy metric spaces and fuzzy topologies.

One of the possible formulations of the limit of a sequence in a metric space $(X, d)$ might be given as follows:

$$
\begin{aligned}
& \lim x_{n}=l \Longleftrightarrow \\
& \inf \left\{\varepsilon: \varepsilon>0, \exists n_{\varepsilon} \in N \ni \forall n \geq n_{\varepsilon}, d\left(x_{n}, l\right)<\varepsilon\right\}=0,
\end{aligned}
$$

where $x_{n}$ is a sequence in $X$.

A many-valued counterpart of this relation is given in [18, 21] for the definition of similarity-based fuzzy limits.

Denoting the set $\left\{\varepsilon: \varepsilon>0, \exists n_{\varepsilon} \in \mathbb{N} \ni \forall n \geq n_{\varepsilon}, d\left(x_{\mathcal{\varepsilon}}, l\right)<\varepsilon\right\}$ by $A_{x_{n},}$,

$$
\widetilde{\lim }\left(x_{n}, l\right)=E\left(0, \inf A_{x_{n}, l}\right), \quad \forall l \in X .
$$

Later in the same works the authors prove that similaritybased fuzzy limits coincide with limit maps of a specific fuzzy topology on $X$.

At this point we aim to establish a connection between similarity-based fuzzy metrics and $L$-topologies via similarity-based fuzzy limits; to do this let us first briefly recall some concepts in $L$-topologies.

Let $X$ be a nonempty set and $(L, \leq, \otimes)$ complete quasimonodial lattice; a subfamily $\tau$ of $L^{X}$ satisfying the following properties is called $L$-topology on $X$ and is given by Höhle and Šostak in [22]:

(T1) $1_{X} \in \tau$ and $1_{\varnothing} \in \tau$,

(T2) $f_{1}, f_{2} \in \tau \Rightarrow f_{1} \otimes f_{2} \in \tau$,

(T3) $\forall i \in K, f_{i} \in \tau \Rightarrow \bigvee_{i \in K} f_{i} \in \tau$, where $K$ is any index set.

In this definition, choosing $L=[0,1]$ and the connective $\otimes$ as the $\wedge t$-norm remains a special case which is named Chang's [23] fuzzy topology in former literature but later on called the $[0,1]$-topology on $X$ (where $\otimes$ is not restricted to being the minimum). From now on by $[0,1]$-topology we mean the case where $L=[0,1]$ and $\otimes=\wedge$.

Starting with a set $X$ and a $*$-similarity $E$ on $X$ it is always possible to obtain a $[0,1]$-topology on $X$ as shown below. if

Let $X$ be a set and $E$ a similarity on $X$ and $f: X \rightarrow[0,1]$;

$$
f(x) * E(x, y) \leq f(y), \quad \forall x, y \in X,
$$

then $f$ is called $E$-extensional. 
The set of all $E$-extensional maps denoted by $\Gamma(X, E)$ is a $[0,1]$-topology on $X[24]$.

In $[18,21]$ the authors have shown that $\widetilde{\lim }\left(x_{n}, l\right)$ coincides with the limit map [22] on $\Gamma\left(X, F_{E, d}\right)$, where $E$ is a *equality on $\mathbb{R}$ invariant under + and compatible with $\leq$ and $F_{E, d}$ is a $*$-similarity relation on $X$ defined by $F_{E, d}=$ $E(0, d(x, y)), \forall x, y \in X$.

In the following proposition we construct a similaritybased fuzzy limit by the mean of a similarity-based fuzzy metric.

Proposition 15. Let $(X, d)$ be a metric space, $E$ a *equality on $\mathbb{R}$ compatible with $\leq$, invariant under + , and $\preccurlyeq$ the corresponding E-ordering on $\mathbb{R}$. For a sequence $\left(x_{n}\right)$ in $X$ the fuzzy relation $\overparen{\lim }\left(x_{n}, l\right)$ defined by

$$
\widetilde{\lim }\left(x_{n}, l\right)=\delta_{E}\left(0, \inf A_{x_{n}, l}\right), \quad \forall l \in X, \forall\left\{x_{n}\right\} \subset X,
$$

is a similarity-based fuzzy limit on $X$.

Corollary 16. Let $(X, d)$ be a metric space, $E$ a $*$-equality on $\mathbb{R}$ compatible with $\leq$, invariant under + , and $\preccurlyeq$ the corresponding E-ordering on $\mathbb{R}$; then $\delta_{E}\left(0, \inf A_{x_{n}, l}\right)$ coincides with the limit map on $\Gamma\left(X, F_{E, d}\right)$.

By this corollary $\Gamma\left(X, F_{E, d}\right)$ can be considered as the fuzzy topology induced by the similarity-based fuzzy metric $\left(X, E, \widetilde{\preccurlyeq}, \delta_{E}\right)$.

\section{Conclusions and Future Works}

We have developed a new version of fuzzy metrics by making use of similarity relations. We have shown the relation between our metrics and former ones and we also have proposed some easy constructions of them. Nevertheless the more important thing we observed was the connection of similarity-based fuzzy metrics with fuzzy topologies. This connection yields to the conclusion that the new fuzzy metric seems to fit well in a complete theory, namely, the similaritybased real line, that is the main motivation behind this work and is encouraging further works in this direction. Towards the similarity-based fuzzy real line some concepts already exist in the literature. Among these in this paper we have mentioned similarity-based orderings and similarity-based fuzzy limits; another example is the similarity-based positive class [25]. The next step might immediately be the absolute value and trying to complete the structure, it follows naturally that similarity-based continuity and differentiation will lie at the heart of other future works.

\section{Competing Interests}

Hereby the authors declare that there is no person with conflict of interest regarding the publication of this paper.

\section{References}

[1] M. M. Fréchet, "Sur quelques points du calcul fonctionnel," Rendiconti del Circolo Matematico di Palermo, vol. 22, no. 1, pp. 1-72, 1906.
[2] K. Menger, "Statistical metrics," Proceedings of the National Academy of Sciences of the United States of America, vol. 28, pp. 535-537, 1942.

[3] A. Wald, "On a statistical generalization of metric spaces," Proceedings of the National Academy of Sciences of the United States of America, vol. 29, pp. 196-197, 1943.

[4] B. Schweizer and A. Sklar, "Statistical metric spaces," Pacific Journal of Mathematics, vol. 10, pp. 313-334, 1960.

[5] I. Kramosil and J. Michalek, "Fuzzy metrics and statistical metric spaces," Kybernetika, vol. 5, pp. 336-344, 1975.

[6] A. George and P. Veeramani, "On some results in fuzzy metric spaces," Fuzzy Sets and Systems, vol. 64, no. 3, pp. 395-399, 1994.

[7] V. Gregori and S. Romaguera, "Some properties of fuzzy metric spaces," Fuzzy Sets and Systems, vol. 115, no. 3, pp. 485-489, 2000.

[8] O. Kaleva and S. Seikkala, "On fuzzy metric spaces," Fuzzy Sets and Systems, vol. 12, no. 3, pp. 215-229, 1984.

[9] V. Novăk, Fuzzy Sets and Their Applications, Adam Hilger, Bristol, UK, 1989.

[10] L. A. Zadeh, "Fuzzy sets," Information and Control, vol. 8, no. 3, pp. 338-353, 1965.

[11] D. Dubois and H. Prade, "Operations on fuzzy numbers," International Journal of Systems Science, vol. 9, no. 6, pp. 613626, 1978.

[12] U. Höhle and N. Blanchard, "Partial ordering in $L$ underdeterminate sets," Information Sciences, vol. 35, no. 2, pp. 133-144, 1985.

[13] R. Bělohlávek, Fuzzy Relational Systems: Foundations and Principles, Kluwer Academic/Plenum Press, New York, NY, USA, 2002.

[14] U. Bodenhofer, "A similarity-based generalization of fuzzy orderings preserving the classical axioms," International Journal of Uncertainty, Fuzziness and Knowledge-Based Systems, vol. 8, no. 5, pp. 593-610, 2000.

[15] U. Bodenhofer, "Representations and constructions of similarity-based fuzzy orderings," Fuzzy Sets and Systems, vol. 137, no. 1, pp. 113-136, 2003.

[16] L. A. Zadeh, "The concept of a linguistic variable and its application to approximate reasoning-I," Information Sciences, vol. 8, no. 3, pp. 199-249, 1975.

[17] M. Mizumoto and K. Tanaka, "Some properties of fuzzy numbers," in Advances in Fuzzy Set Theory and Applications, pp. 153-164, North-Holland, New York, NY, USA, 1979.

[18] G. Soylu, On the vague real line [Ph.D. thesis], Akdeniz Üniversitesi, 2008.

[19] J. L. Kelley, General Topology, Springer, New York, NY, USA, 1975.

[20] L. Valverde, "On the structure of F-indistinguishability operators," Fuzzy Sets and Systems, vol. 17, no. 3, pp. 313-328, 1985.

[21] G. Soylu, "Similarity-based fuzzy limits," Fuzzy Sets and Systems, vol. 159, no. 24, pp. 3380-3387, 2008.

[22] U. Höhle and A. P. Šostak, "Axiomatic foundations of fixed-basis fuzzy topology," in Mathematics of Fuzzy Sets: Logic, Topology, and Measure Theory, U. Höhle and S. E. Rodabaugh, Eds., vol. 3 of The Handbooks of Fuzzy Sets Series, pp. 123-272, Kluwer Academic Publishers, Boston, Mass, USA, 1999. 
[23] C. L. Chang, "Fuzzy topological spaces," Journal of Mathematical Analysis and Applications, vol. 24, pp. 182-190, 1968.

[24] U. Höhle, "Topological aspects of non-convergent sequences: a comment on Burginfis concept of fuzzy limits," Fuzzy Sets and Systems, vol. 149, no. 3, pp. 399-412, 2005.

[25] G. Soylu, "E-vague positive class," International Journal of Uncertainty, Fuzziness and Knowledge-Based Systems, vol. 17, no. 1, pp. 137-146, 2009. 


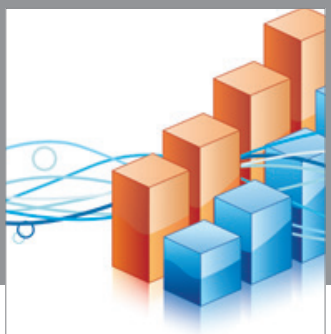

Advances in

Operations Research

vatem alat4

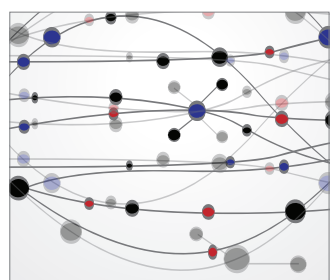

\section{The Scientific} World Journal
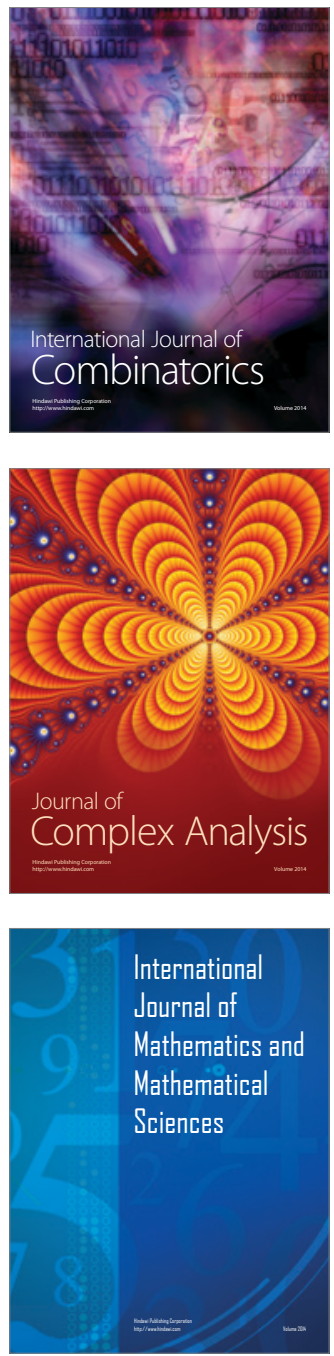
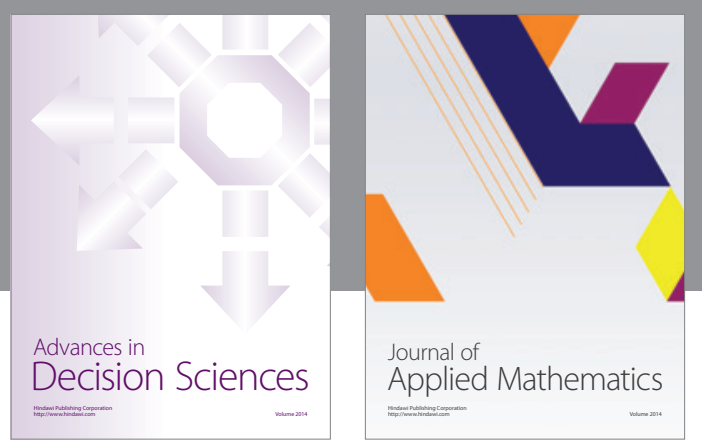

Algebra

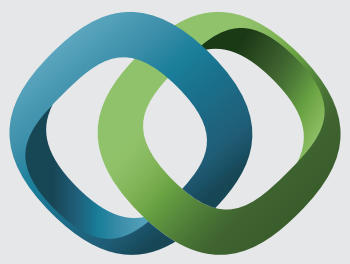

\section{Hindawi}

Submit your manuscripts at

http://www.hindawi.com
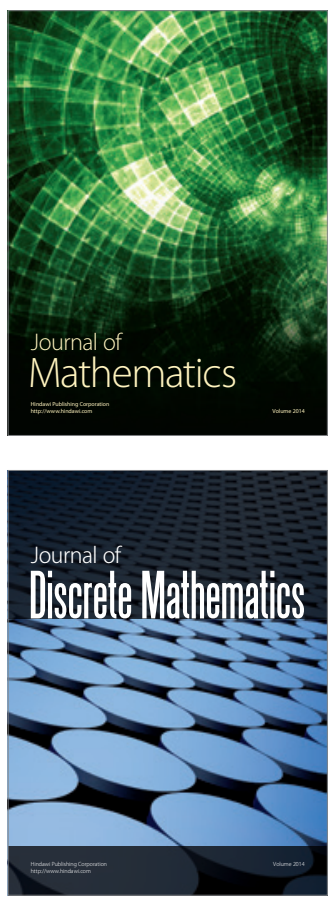

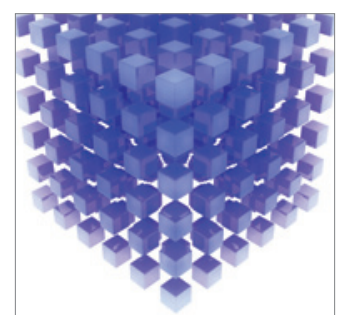

Mathematical Problems in Engineering
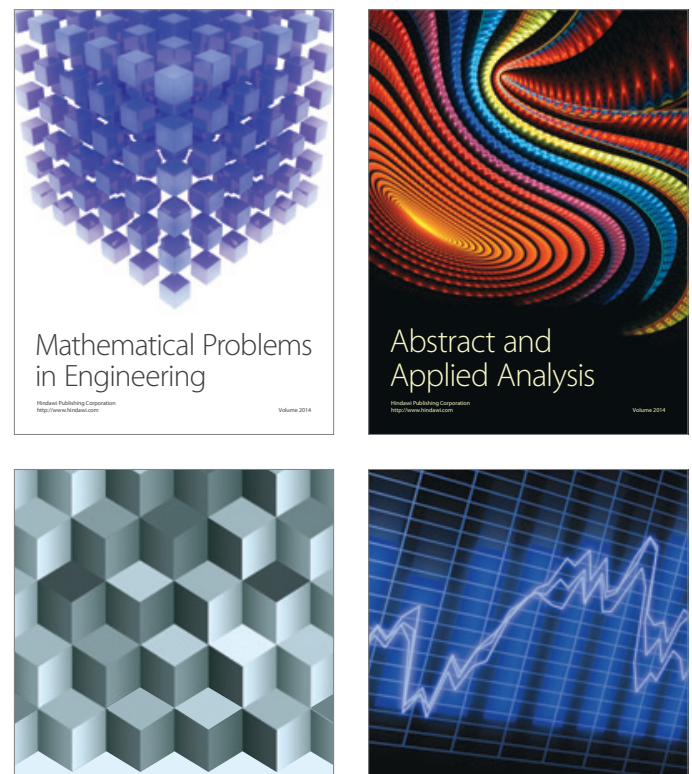

Journal of

Function Spaces

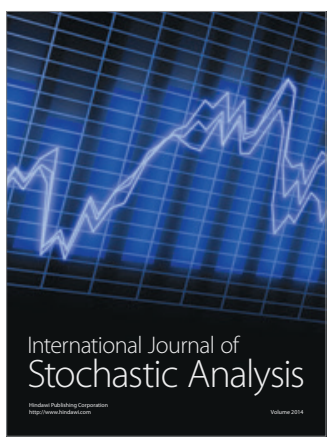

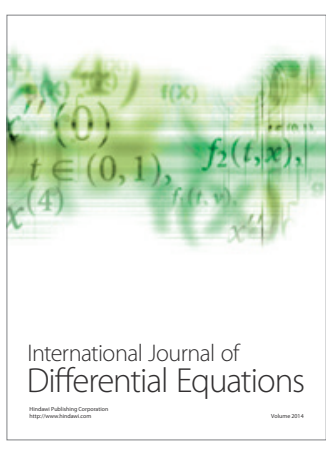
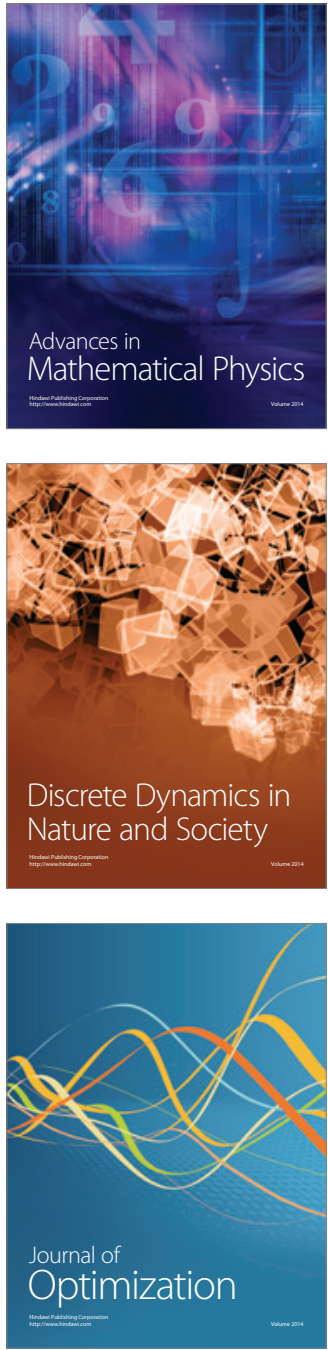